\section{Trends in the development of MET inhibitors for hepatocellular carcinoma}

\author{
Hitomi S Okuma' \& Shunsuke Kondo*,1
}

\begin{abstract}
Hepatocellular carcinoma is the third most common cause of cancer-related deaths worldwide. The multikinase inhibitor sorafenib has improved survival and is now considered the standard of care; however, the benefits are still disappointing, and thus, new effective treatments are required. In human hepatocellular carcinoma, MET, which is encoded by the HGFR gene, is activated by amplification, overexpression or mutation, and it has recently emerged as a possible therapeutic target in various tumors including hepatocellular carcinoma. In fact, some drugs targeting the HGF/MET axis are currently under investigation in clinical trials. Here, we review the role of MET and trends in the development of MET inhibitors for hepatocellular carcinoma.
\end{abstract}

First draft submitted: 19 October 2015; Accepted for publication: 7 January 2016; Published online: 17 March 2016

Hepatocellular carcinoma (HCC) is the third most common cause of cancer-related deaths worldwide [1], and is the most widespread in southeast Asia and sub-Saharan Africa. In western countries, both HCC morbidity and mortality rates have increased over the past 40 years [2]. Conversely, in Japan, these rates have decreased [3]. This discordance is believed to result from differences in the prevalence of hepatitis viruses in the regions, as well as the timing of the spread of viral infection. Based on previous studies, hepatitis $\mathrm{C}$ virus first began to infect large numbers of young adults in Japan, southern Europe and North America [4].

The causes of HCC include hepatitis B virus, excessive alcohol consumption, obesity, diabetes mellitus and nonalcoholic fatty liver disease [5]. The current treatments for HCC include surgical resection, liver transplantation and ablation for curable tumors, and a 5-year survival rate over $50 \%$ can be achieved using these approaches [6]. In asymptomatic patients with multifocal disease without vascular invasion or extrahepatic spread and who are not candidates for curative treatment, chemoembolization and sorafenib [7] have both improved survival. In general, HCCs are considered chemoresistant; therefore, targeted molecular therapies are a highly active area of research.

The multikinase inhibitor sorafenib (active against the $c-R A F$ and $b-R A F$ proto-oncogenes, serine/threonine kinases, VEGFR, KIT and PDGFR- $\beta$ ) has been shown to improve survival compared with other available treatments, and is now the standard of care [7]. However, the benefits are still disappointing, and new effective treatments are required. Although many studies having identified genes and/or pathways that are involved in the tumorigenesis of HCC, unlike in other solid tumors, no genes to which HCC cells are 'addicted' have been identified. The HGFR, namely MET which is a tyrosine kinase encoded by the $\mathrm{N}$-methyl- $\mathrm{N}^{\prime}$-nitrosoguanidine human osteosarcoma transforming gene, has emerged as a possible therapeutic target in various tumors including HCC [8]. Here, we

'Department of Experimental Therapeutics, National Cancer Center, Tokyo, Japan

*Author for correspondence: Tel.: +81 335422 511; Fax: +81 335423 815; shkondo@ncc.go.jp

\section{KEYWORDS}

- biomarkers • clinical trials

- hepatocellular carcinoma

- HGF • MET 
review of the role of MET in HCC and trends in the development of MET inhibitors for HCC.

\section{The role of MET in tumor biology}

The MET proto-oncogene encodes the tyrosine kinase receptor for HGF. Upon binding of HGF to MET (Figure 1), MET is activated and signals through the RAS/MAPK and PI3K/AKT pathways thereby promoting several biological activities including cell proliferation, invasion, protection from apoptosis and angiogenesis (Figure 2). Therefore, in addition to the crucial role of MET signaling in liver development and regeneration, mutations or alterations in $M E T$ can lead to carcinogenesis $[9,10]$. The connection between MET and cancer development was supported by the identification of three germline mutations in the $M E T$ gene in hereditary papillary renal carcinoma; this missense mutation resulted in constitutive activation of MET in a ligand-independent manner and tumorigenesis [11].

Phosphorylation and activation of MET can also occur in the absence of HGF through crosstalk with other membrane receptors such as cluster of differentiation 44 (CD44) and $\alpha 6 \beta 4$ integrin, the FAS cell surface death receptor, Plexin $\mathrm{B}$, or receptor tyrosine kinases such as the ERBB family members (EGFR, HER2) and VEGFR [12-16]. These signaling networks are

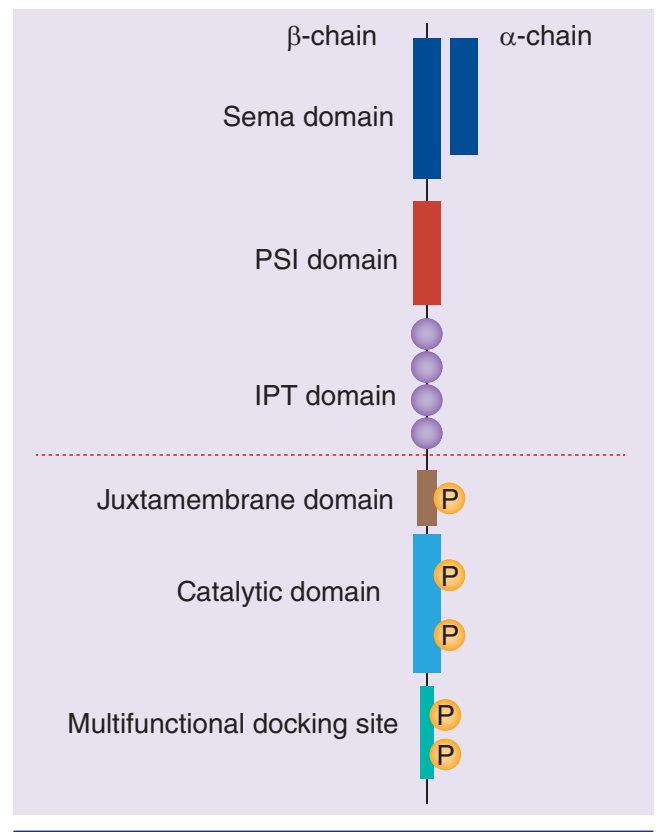

Figure 1. The structure of the MET receptor. IPT: Immunoglobulin-plexin transcription; PSI: Plexin-semaphorin-integrin;

Sema: Semaphorin. thought to cooperate to promote tumorigenesis and/or metastasis, and to induce resistance to targeted molecular therapies.

Deregulated miRNAs have also been found to play crucial roles in the initiation and progression of HCC. Many miRNAs have been shown to regulate the expression of proteins in tyrosine kinase receptors, including miRNA-119a-3p [17], miRNA-34a [18] and miRNA-93 [19] to name a few. Restoring the expression of miRNA119a-3p in HCC cells which regulates MET and mTOR [17] led to G1 arrest, reduced invasive capability, enhanced susceptibility to hypoxia and increased sensitivity to doxorubicin-induced apoptosis. MiRNA-34a downregulated MET expression and inhibited migration and invasion of HCC cells [18]. MiRNA-93 enhanced the MET/PI3K/AKT pathway by inhibiting PTEN and CDKN1A [19].

Aberrant MET expression has been demonstrated in many kinds of solid tumors in vitro and has been correlated with poor prognosis [20]. In lung cancer cell lines, both HGF and MET are expressed and create an autocrine loop [21,22]. In a previous study, high HGF expression was associated with poor prognosis, and MET overexpression was associated with resistance to EGFR tyrosine kinase inhibitors in a gefitinib-sensitive lung cancer cell line [23]. These findings led to clinical trials in which agents that could block both MET and EGFR activity were evaluated. For example, a Phase II trial of a selective small-molecule MET inhibitor, tivantinib, combined with erlotinib resulted in prolonged progression-free survival (PFS) [24] which led to a Phase III trial, the MARQUEE trial that also showed a survival benefit with the combination arm [25]. Other trials investigating small-molecule inhibitors and monoclonal antibodies that target MET in combination with EGFR tyrosine kinase inhibitor (TKI) therapy are ongoing. In colorectal cancer, it was hypothesized that the addition of a MET inhibitor could decrease resistance to the anti-EGFR monoclonal antibody cetuximab. Indeed, a Phase I/II trial of tivantinib in combination with irinotecan and cetuximab demonstrated favorable clinical response particularly in MET-high tumors [26].

\section{Preclinical studies of HGF/MET \& hepatocellular carcinoma in vivo \& in vitro} HGF was originally identified as having cytoprotective effects on the liver against tissue damage; 
these effects were revealed by the mitogenic and motogenic activities of HGF, and its ability to suppress apoptotic cell death [27]. Studies have also shown that HGF and MET provide essential signals that promote the survival and proliferation of hepatocytes during embryogenesis $[11,28]$. These critical roles in liver development and regeneration have led to additional studies that aimed to identify genetic and functional alterations of the HGF/MET pathway in HCC.

The mechanisms underlying the effects of HGF stimulation and MET inhibition in HCC remain unclear, as preclinical data show contradicting results on the expression and role of HGF and MET. In preclinical studies, the loss of MET signaling in hepatocytes increased rather than inhibited tumor initiation $[29,30]$. In contrast, another study demonstrated a protumorigenic role for $M E T$ in $M E T$ transgenic or $M E T$-transfected mice [31], and downregulation or inhibition of MET demonstrated to interfere with both cell growth and invasion [32-34]. Studies of $H G F$ transgenic mice have revealed a potential protumorigenic role of HGF in HCC. In these studies, $33 \%$ of male and $23 \%$ of female transgenic mice, but no wild-type mice developed HCC [35,36]; however, the results of other studies have not supported a HCC-inducing role of HGF in human $H G F$ cDNA transgenic mice [37]. Studies showed that HGF inhibited HCC cell growth in vitro $[38,39]$. Some reasons of the contradicting results may be explained by the use of different techniques, the lack of an agreed scoring system and the small sample sizes of the studies [40]. In another study that used a genetically altered mouse model, human MET was overexpressed in liver cells, which led to the development of HCC. Since human MET cannot bind murine HGF, the activation of MET could only have occurred through a ligand-independent mechanism. Two studies identified another protein, des-gamma-carboxy prothrombin, which also binds to MET and induces both proliferation of HCC cell lines and JAK/STAT3 activation [41]. These results have suggested an oncogenic role of the MET pathway in HCC and led to the hypothesis that overactivation of MET could cause HCC. The mechanism by which the receptor is activated in HCC is not completely understood. Nevertheless, most studies agree on the decrease of HGF in HCC and that MET activation is largely HGF-independent in HCC upon its role as an oncogene.

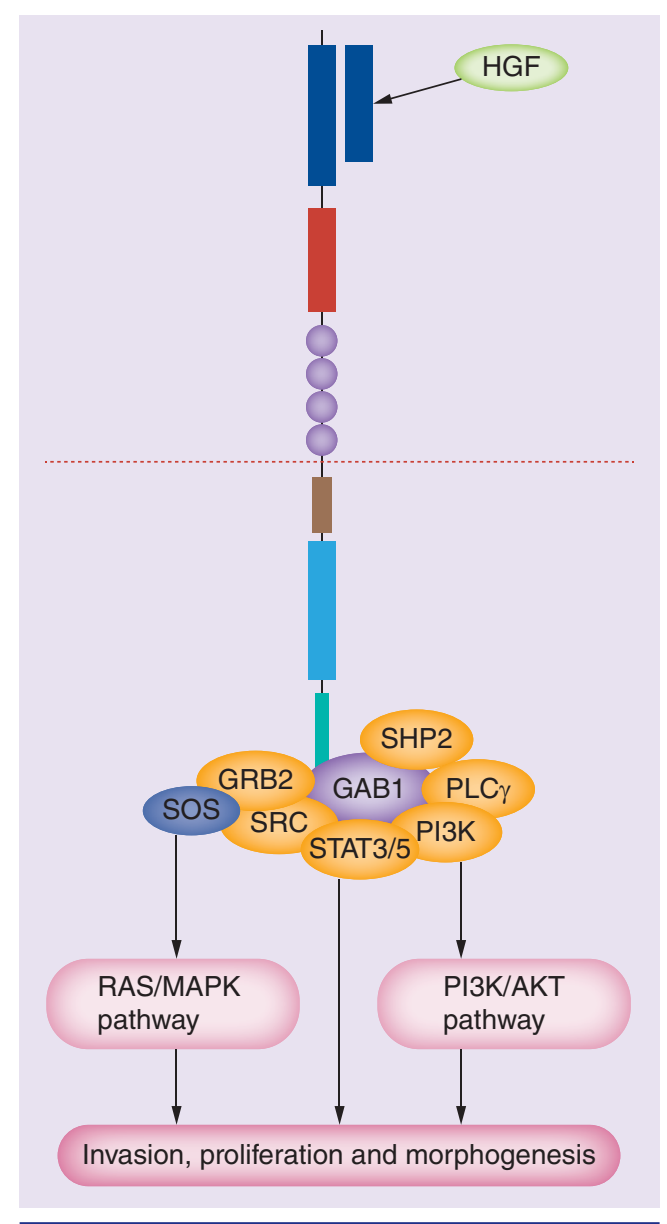

Figure 2. The MET signaling network.

\section{HGF/MET \& human HCC}

Similar to the preclinical data mentioned above, clinical data on the role of HGF and MET in HCC are also conflicting. In human tumors, MET can be activated by gene amplification, overexpression or activating mutations. According to a recent study, MET amplification was only observed in one out of 59 cases whereas aneuploidy of chromosome 7 (the location of both the MET and $H G F$ genes) was observed in 22 of 59 patients [42]. Another study identified MET but not $H G F$ as one of ten genes in the amplification peak located at $7 \mathrm{q} 31.2$, which was present in 4-5\% of cases of HCC [43], while one study did not identify any MET-activating mutations in $24 \mathrm{HCC}$ patients [44].

In a previous study that investigated the correlation between MET/HGF expression and clinical data, increases in MET mRNA and protein expression were observed in 30-40\% of HCCs by northern blot analysis and immunohistochemical (IHC) staining. A correlation 
between poor-to-moderate differentiation of the cancer cells and MET overexpression was noted [45]. In a study using PCR, increased MET expression was observed in some HCCs, whereas HGF was expressed at lower levels in HCC tissue than in peritumoral tissue [46]. Using western blot analysis, MET overexpression was observed in $48 \%$ of HCC patients, and these patients had a significantly shorter 5-year survival rate compared with patients with low MET expression (33.5 vs 80.3\%; p < 0.05); however, this study failed to demonstrate a significant relationship between HGF expression and clinical performance [47].

In addition, quantitative reverse transcriptionPCR of 15 HCC and peritumoral tissue samples revealed low levels of HGF in highly differentiated tumors and overexpression of MET in both poorly differentiated tumors and early recurrent tumors. Although the tissue HGF levels provided little prognostic information, plasma HGF levels were consistently higher in patients with HCC compared with normal subjects, and circulating HGF levels correlated with decreased overall survival [48]. On the other hand, it should be noted that elevated circulating HGF levels have also been observed in patients with hepatitis and liver cirrhosis.

The above findings have led to difficulties in characterizing the role(s) of HGF/MET as predictive biomarkers in patients with HCCs. The predictive potential of $\mathrm{HGF}$ remains unclear based on current data and additional prospective studies are required. Amplification or mutation of $M E T$ appears to be quite rare; therefore, MET overexpression as measured by IHC or reverse transcriptase-PCR is probably the only criterion for evaluating a possible response to anti-MET drugs, although no standardized tests have been developed thus far.

\section{Agents that target the MET signaling pathway}

Currently, there are no approved drugs that target the MET signaling pathway in HCC. Initial attempts to identify MET inhibitors began with the discovery of the microbial alkaloid K-252a, the first structure to be solved in complex with the unphosphorylated MET kinase domain [49]. More selective MET inhibitors were subsequently designed such as the firstgeneration drug SU11274 [50] and the secondgeneration drug PHA665752 [51]. PHA665752, a selective inhibitor of MET, has been shown to suppress cell proliferation and induce apoptosis in MET-positive cell lines, but to have no effects on MET-negative cells. This was also demonstrated in xenograft nude mice models where MET inhibition was capable of significantly inhibiting MET-positive HCC tumors [52]. These discoveries suggested that MET was a promising target for the treatment of HCC and that overexpression of MET may be a predictive biomarker for therapeutic response. Currently, several drugs targeting the HGF/MET axis are under clinical investigation (Figure 3). These include monoclonal antibodies against HGF or MET and specific or nonspecific small-molecule MET-TKIs.

\section{Monotherapy \\ - Multikinase-targeted tyrosine kinase inhibitors}

\section{Foretinib (GSK1363089)}

Foretinib was the first MET-TKI to be used in the clinic. It is an oral TKI that targets MET, macrophage stimulating 1 receptor $(\mathrm{RON}), \mathrm{AXL}$ receptor tyrosine kinase, TEK tyrosine kinase endothelial (TIE-2) and VEGFR. In a Phase I/II study of Asian patients with HCC, the objective response rate (ORR) was 24\% (95\% CI: $11-40 \%)$, the disease control rate (DCR) was 79\% (95\% CI: 63-90\%) and the median time to progression (TTP) was 4.2 months $(95 \% \mathrm{CI}$ : 2.7-7.5 months) among the 38 patients who were evaluated, and had acceptable safety margin and tolerability [53].

\section{Cabozantinib (XL184)}

Cabozantinib is an oral TKI that targets MET, the RET proto-oncogene and VEGFR-2. In a Phase II study of $41 \mathrm{HCC}$ patients who were treated with $100 \mathrm{mg}$ cabozantinib once daily during a 12-week lead-in stage, three of the 36 patients for whom tumor assessment could be performed achieved a partial response (PR). The median PFS was 4.4 months and the median overall survival (OS) was 15.1 months. $50 \%$ of the population received the treatment as second-line, and the rest was as first-line. The PFS did not differ between patients with or without prior use of sorafenib [54]. Based on these promising results, a Phase III randomized, double-blind study was designed to further evaluate the efficacy of cabozantinib compared with placebo in advanced HCC patients with prior sorafenib treatment, which is ongoing (NCT01908426). 


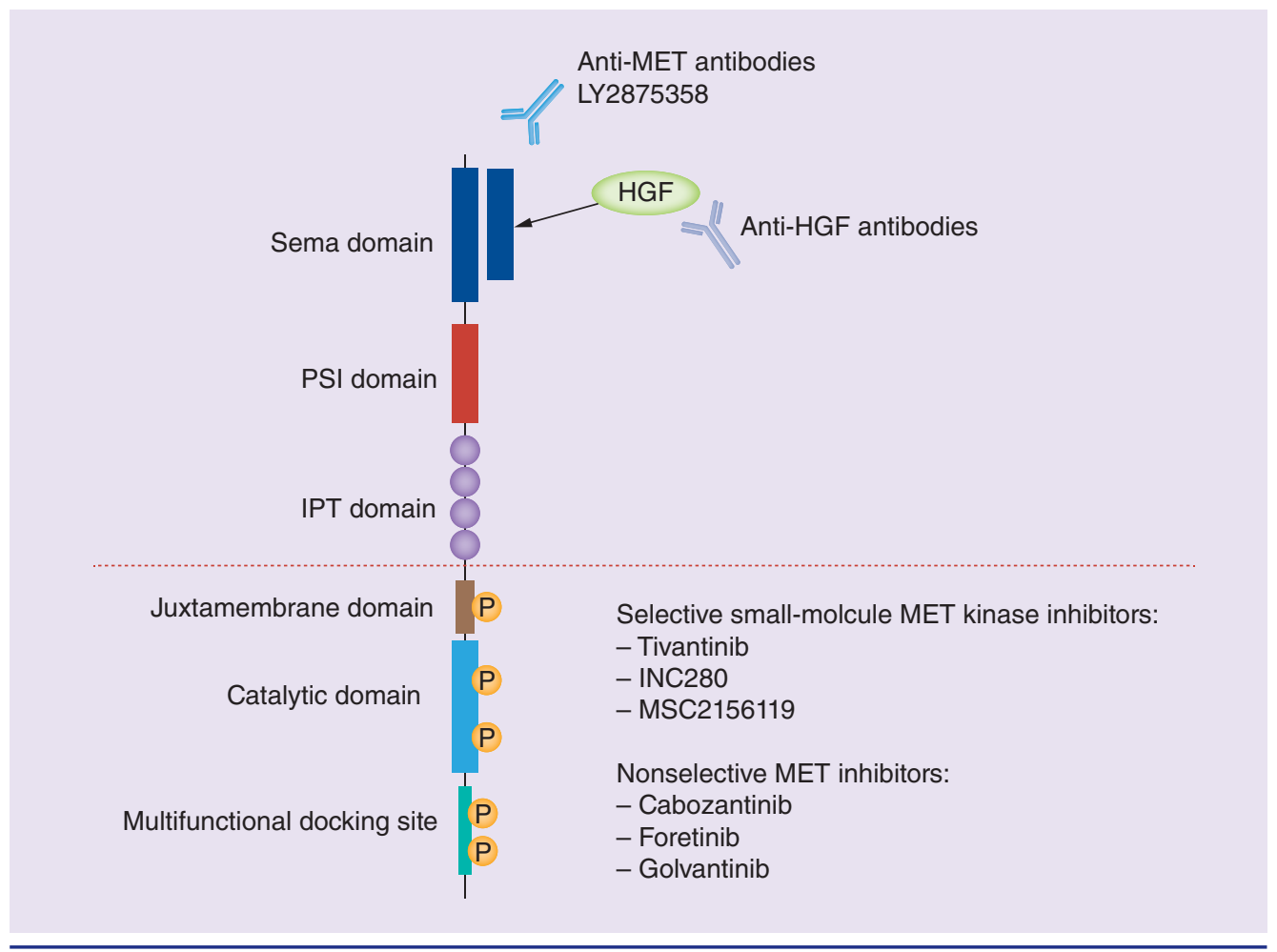

Figure 3. MET as a clinical target in hepatocellular carcinoma.

IPT: Immunoglobulin-plexin transcription; PSI: Plexin-semaphorin-integrin; Sema: Semaphorin.

- Monoclonal antibodies

LY2875358

LY2875358 is a humanized immunoglobulin G4 monoclonal bivalent antibody that binds to and inhibits ligand-dependent and ligandindependent activation of MET. A Phase I (part A) dose-escalating study of 23 patients with advanced solid tumors included one case of HCC. The recommended Phase II dose (RPTD) was $750 \mathrm{mg}$ every 2 weeks and was not associated with dose-limiting toxicities or serious adverse events. Additionally, one patient with papillary urothelial carcinoma achieved PR. The combination therapy of LY2875358 and erlotinib in advanced non-small-cell lung cancer (NSCLC) patients was examined in part B of this study, which will be discussed in a subsequent section of this review [55].

\section{- Selective small-molecule MET tyrosine kinase inhibitors \\ Tivantinib (ARQ197)}

Among the oral small-molecule MET-TKIs in clinical trials for HCC, tivantinib is the most tested and has received the most exposure to date. Tivantinib has been tested as a selective MET inhibitor, although conflicting results remain as to whether the agent is purely 'selective' or not. Exposure to tivantinib resulted in the inhibition of proliferation of MET expressing cancer cell lines followed by a mouse xenograft study, which demonstrated growth inhibition of human tumors upon administration of tivantinib [56]. This was supported by a Phase I proof of mechanism study using serial biopsies [57], in which tivantinib decreased phosphorylated MET, total MET, and phosphorylated focal adhesion kinase and increased terminal deoxynucleotidyl transferase-mediated deoxyuridine triphosphate-biotin nick-end labeling (TUNEL) staining in tumor biopsies of 15 patients. It has shown further promising antitumor activity in clinical studies as a monotherapy and in combination with sorafenib. In a Phase II study [58] of HCC patients with Child-Pugh A cirrhosis who had progressed on, or were unable to tolerate first-line chemotherapy were randomized in a 2:1 fashion to receive tivantinib $360 \mathrm{mg}$ twice daily (b.i.d.) or placebo, resulting in 71 and 36 treated patients, respectively. The dose was adjusted to $240 \mathrm{mg}$ b.i.d. due to grade $\geq 3$ neutropenia. The TTP was found to be longer in the tivantinib arm (1.6 months [95\% CI: $1.4-2.8$ ] vs 1.4 months [95\% CI: 1.4-1.5]; hazard ratio [HR]: 0.64; 
90\% CI: 0.43-0.94; p = 0.04). Eight patients $(21 \%)$ in the $360 \mathrm{mg}$ tivantinib group had grade $\geq 3$ neutropenia compared with two $(6 \%)$ patients in the $240 \mathrm{mg}$ group, and thus the latter dose was favored for further confirmation.

In patients with tumors that showed high MET expression, all of the efficacy parameters favored tivantinib compared with placebo. The median TTP was 2.7 months (95\% CI: 1.4-8.5) for 22 patients with high MET expression who received tivantinib versus 1.4 months $(95 \% \mathrm{CI}$ : 1.4-1.6) for 15 patients with high MET expression who received the placebo (HR: 0.43; 95\% CI: 0.19-0.97; $\mathrm{p}=0.03)$. The median PFS was 2.2 and 1.4 months (HR: $0.45 ; 95 \% \mathrm{CI}$ : $0.21-0.95 ; \mathrm{p}=0.02$ ), the DCR was 50 and $20 \%$, and the median OS was 7.2 and 3.8 months, respectively (HR: 0.38; 95\% CI: 0.18-0.81; $\mathrm{p}=0.01)$. These results suggested that aside from its prognostic role, MET expression was predictive of the response to tivantinib, and that tumor biopsy is required in order to identify patients who may benefit from this therapy. In contrast, two recent studies showed that tivantinib did not inhibit MET activity in a variety of tumors, and that the drug exerted its pharmacologic action in cells that were not dependent on MET for growth and survival, including cells that did not express MET $[59,60]$. This has caused some debates on the 'selective' role of the drug.

Previous population pharmacokinetic analyses have shown that tivantinib reaches higher concentrations in the plasma of patients with HCC compared with patients with other types of tumors. The mean area under the curve for $240 \mathrm{mg}$ tivantinib b.i.d. was $25,660 \mathrm{ng} \cdot \mathrm{h} / \mathrm{ml}$ in patients with HCC compared with $12,050 \mathrm{ng} \cdot \mathrm{h} / \mathrm{ml}$ in NSCLC patients treated with $360 \mathrm{mg}$ of tivantinib b.i.d. As for Japanese patients, in a Phase I pharmacokinetic study, 360 and $240 \mathrm{mg}$ b.i.d. were recommended for patients with solid tumors other than HCC who were CYP2C19-extensive or -poor metabolizers, respectively. In contrast, among HCC patients who received $240 \mathrm{mg}$ b.i.d., five of nine of the extensive metabolizers developed neutropenia-related dose-limiting toxicities that accompanied plasma tivantinib concentrations, a ratio that was higher than was expected based on previous studies. This caused the investigators to discontinue the dose at $240 \mathrm{mg}$ and conclude that $120 \mathrm{mg}$ was recommended for Japanese patients with HCC regardless of CYP2C19 phenotype [61].

Based on the promising results of the Phase II trial, Phase III studies including the
METIV-HCC study performed in western countries (NCT01755767) and the JET-HCC study in Japan (NCT02029157) were initiated and are ongoing. In the METIV-HCC Phase III trial, the original planned dose was $240 \mathrm{mg}$ b.i.d., which was determined based on previous trials; however, the dose was reduced to $120 \mathrm{mg}$ b.i.d. following double exposure to the drug caused by the tablet formulation and the increased incidence of neutropenia-related adverse events. On the other hand, JET-HCC was planned at a dosage of $120 \mathrm{mg}$ b.i.d. from the start. All patients with confirmed MET overexpression, defined as $\geq 50 \%$ of tumor cells having a membrane staining intensity of $\geq 2+$ for MET as assessed by IHC in a central laboratory, will be randomized in a 2:1 fashion to either tivantinib or placebo. If the primary end point of improved OS is achieved in the Phase III trials, treatment with tivantinib will provide a rational alternative to best supportive care in patients with MET-overexpressed HCC who experienced disease progression on, or intolerance to, first-line sorafenib therapy. However, we must be careful when applying this biomarker for treatment with tivantinib because of the negative trials on its role as a 'selective' MET inhibitor.

\section{Capmatinib (INC280)}

Capmatinib is a highly selective, oral small-molecule inhibitor of MET that has been shown to be both safe and tolerable in a Phase I dose-escalation trial that included 33 patients with heavily treated solid tumors [62], of whom HCC patients represented the most frequently enrolled population (45\%). The RPTD was $600 \mathrm{mg}$ b.i.d. This inhibitor also showed clinical efficacy, with a DCR of 24\%. Phase II studies of HCC (NCT01737827) and various other solid tumors are ongoing.

\section{- MSC2156119J (EMD 1214063)}

MSC2156119J is a highly selective oral inhibitor of MET. A Phase I dose-escalation study of 100 patients with advanced solid tumors showed that the inhibitor had promising antitumor activity, and the RPTD was determined to be $500 \mathrm{mg}$ per day [63]. This result has led to an ongoing Phase Ib/II trial comparing MSC2156119J monotherapy in first-line treatment versus sorafenib in Asian patients with MET-positive advanced HCC (NCT01988493). In the European centers, another Phase Ib/II single arm trial with monotherapy 300-500 mg every $24 \mathrm{~h}$ in 21-day cycles is ongoing in the MET-positive HCC population (NCT02115373). 
Combination therapy

- MET \& VEGF: crosstalk \& resistance to anti-VEGF(R) agents

Several in vivo and in vitro studies have supported the association between HGF/MET and angiogenesis. HGF is also known for its function as an angiogenic factor that promotes endothelial cell motility and growth [64]. Recently, it was shown that VEGF directly regulated tumor cell invasion through promoting recruitment of the protein tyrosine phosphatase $1 \mathrm{~B}$ to a MET/VEGFR2 heterocomplex and suppression of HGF-dependent MET phosphorylation and tumor cell migration. Therefore, when VEGF was blocked (e.g., by bevacizumab), increased MET activity was restored in a hypoxia-independent manner [15]. Antiangiogenic therapies including sorafenib can also induce hypoxia, resulting in an increase in hypoxia-inducible factor-1 $\alpha$ expression, which is known to induce MET transcription and expression [65,66]. These findings suggest that while antiangiogenic molecules typically act as inhibitors of tumor growth by suppressing angiogenesis, these proteins can also promote aggressive behavior of the tumors. Thus, simultaneous inhibition of the MET and VEGFR pathways in HCC is currently under investigation.

\section{Tivantinib (ARQ197) plus sorafenib}

A Phase I dose-escalating study of tivantinib in combination with sorafenib for the treatment of advanced solid tumors was recently performed [67]. The RPTDs were $360 \mathrm{mg}$ b.i.d. for tivantinib and $400 \mathrm{mg}$ b.i.d. for sorafenib for all cancer histologies except HCC, where the corresponding values were 240 and $400 \mathrm{mg}$ b.i.d. for tivantinib and sorafenib, respectively. For the $20 \mathrm{HCC}$ patients enrolled in the study, the ORR was $10 \%$ and the DCR was $65 \%$, and patients who progressed on sorafenib and were subsequently treated with sorafenib and tivantinib obtained PRs and a complete response. Thus, these preliminary data demonstrated the therapeutic potential of simultaneous inhibition of the MET and VEGFR pathways, including the clinical benefit of MET inhibition for those who may have gained a MET escape pathway after resistance to anti-VEGFR, sorafenib.

\section{Golvatinib (E7050) plus sorafenib}

Golvatinib is an oral, small-molecule, ATPcompetitive TKI that targets MET, multiple members of the Eph receptor family, KIT and
RON. In an ongoing Phase I/II trial that includes patients with advanced HCC, 13 patients have been enrolled and the maximum tolerated doses of golvatinib and sorafenib were determined to be $200 \mathrm{mg}$ once daily and $400 \mathrm{mg}$ b.i.d., respectively; the PR rate was $17 \%$ and the disease control rate was $45 \%$ for these patients [68]. The Phase II part of the trial is ongoing (NCT01271504).

\section{Golvatinib (E7050) plus lenvatinib}

Lenvatinib is a TKI of VEGFR1-3, FGFRs 1-4, PDGFR- $\alpha$, RET and KIT. In thyroid and endometrial cancer models, golvatinib and lenvatinib inhibited pericyte network formation and Tie2expressing macrophage infiltration resulting in severe perfusion disorder and massive apoptosis [69]. A Phase Ib/II trial of golvatinib and lenvatinib combination therapy in patients with advanced solid tumors is ongoing (NCT01433991).

\section{- MET \& EGFR: crosstalk \& anti-EGFR agent resistance}

In addition to the aforementioned cross-talk between MET and EGFR, amplification of $M E T$ and $H G F$ has been observed in tumors with acquired resistance to EGFR-TKIs including to erlotinib and gefitinib. Therefore, studies of combination therapies that target both MET and EGFR are currently being performed, primarily in patients with lung cancers. The combination therapy of tivantinib and erlotinib was studied in a Phase III trial, the MARQUEE study [25] in patients with wild-type EGFR nonsquamous NSCLC patients. Although the trial was discontinued for futility at the preplanned interim analysis, the median PFS significantly favored adjunct tivantinib as compared with erlotinib monotherapy (3.6 vs 1.9 months, respectively (HR: $0.74 ; \mathrm{p}<0.0001)$ and one of the predetermined secondary end points suggested OS improvement in patients with high MET expression (HR: 0.70; 95\% CI: 0.48-1.01) [70]. The ORR was $10.3 \%$ in the combination arm compared with $6.5 \%$ in the placebo/erlotinib arm $(p<0.05)$, showing promising biological activity. LY2875358, which is a bivalent MET antibody, was evaluated in a Phase I study as a monotherapy (part A) and in combination with erlotinib (part B). The results of part A were mentioned earlier in this review. Part B included 14 NSCLC patients. The RPTD was $750 \mathrm{mg}$ every 2 weeks, and two out of 13 evaluable patients achieved PR. Both patients were positive for MET protein expression. 
MET as a biomarker for HCC

MET overexpression is currently the most plausible biomarker based on preclinical and clinical data, although contradicting opinions remain. Preclinical data have failed to show a consistent relationship between HGF levels and HCC prognosis or predicted response to treatment. The results from the Phase II tivantinib study in HCC patients [58] provided evidence that supported the preclinical and early clinical studies which suggest MET as a predictive biomarker, and late phase studies in other tumor types have also supported this association [70,71].

Although the clinical data and the majority of preclinical data results suggest that MET expression could be a predictive marker for the response to treatment, two recent studies have questioned the mechanism of action of tivantinib [59,60]. These two studies showed that tivantinib does inhibit MET activity, but that the drug also exerted its pharmacologic action in cells that were not dependent on MET for growth and survival, including cells that did not express MET. After concluding that tivantinib may not be 'highly selective MET inhibitor,' the authors instead suggested that the drug acted as a cytotoxic chemotherapy that perturbed microtubule dynamics similar to vincristine, rather than as a targeted agent. In the past, other targeted drugs have also turned out to be cytotoxic including, for example, iniparib, which was intended as a PARP inhibitor but in the end was determined to be a cytotoxic agent $[72,73]$. Nonetheless, regardless of the potential cytotoxic activity that tivantinib may have, most published data support the evidence that tivantinib binds to MET and inhibits it in vitro, in vivo and in patients [74,75].

Collectively, these preclinical and clinical studies have suggested that MET overexpression is a potential pharmacodynamic biomarker, although we must be careful when attempting to translate the preclinical results into the clinic. We are still

\section{Table 1. Ongoing trials of HGF/MET inhibitors in patients with hepatocellular carcinoma.}

\begin{tabular}{|c|c|c|c|}
\hline Therapy & Study type & Subject & Status \\
\hline \multicolumn{4}{|l|}{ Monotherapy } \\
\hline \multicolumn{4}{|l|}{ Tivantinib (ARQ197): } \\
\hline - NCT01755767 & Phase III (vs placebo) & Inoperable HCC with one prior treatment & Ongoing \\
\hline - NCT02029157 (JET-HCC) & Phase III (vs placebo) & $\begin{array}{l}\text { High MET expression; inoperable HCC with one prior sorafenib } \\
\text { treatment }\end{array}$ & Ongoing \\
\hline \multicolumn{4}{|l|}{ Cabozantinib (XL184): } \\
\hline - NCT00940225 & Phase II (vs placebo) & Solid tumors & Completed \\
\hline - NCT01908426 & Phase III (vs placebo) & Advanced HCC with prior sorafenib treatment & Ongoing \\
\hline \multicolumn{4}{|l|}{ Foretinib (GSK1363089): } \\
\hline - NCT00920192 & Phase I & Advanced HCC & Ongoing \\
\hline \multicolumn{4}{|l|}{- INC280 } \\
\hline - NCT01737827 & Phase II & Advanced HCC with MET dysregulation & Ongoing \\
\hline \multicolumn{4}{|l|}{ MSC2156119J (EMD 1214063): } \\
\hline - NCT02115373 & Phase Ib/II & Advanced HCC with prior sorafenib treatment & Ongoing \\
\hline - NCT01988493 & Phase Ib/II (vs sorafenib) & Asian, MET-positive advanced HCC; first-line treatment & Ongoing \\
\hline \multicolumn{4}{|l|}{ Golvatinib (E7050): } \\
\hline - NCT01428141 & Phase I & Advanced solid tumors & Completed \\
\hline \multicolumn{4}{|l|}{ - NCT00921869 } \\
\hline \multicolumn{4}{|l|}{ - NCT00869895 } \\
\hline \multicolumn{4}{|l|}{ LY2875358: } \\
\hline - NCT01287546 & Phase I (Part A) & Advanced cancer & Ongoing \\
\hline \multicolumn{4}{|l|}{ Combination therapy } \\
\hline $\begin{array}{l}\text { Tivantinib (ARQ197) plus sorafenib: } \\
\text { - NCT00827177 }\end{array}$ & Phase I & Advanced solid tumors & Complete \\
\hline $\begin{array}{l}\text { Golvatinib (E7050) plus sorafenib: } \\
\text { - NCT01271504 }\end{array}$ & Phase I/II (vs sorafenib) & HCC; first-line & Ongoing \\
\hline $\begin{array}{l}\text { Golvatinib (E7050) plus lenvatinib: } \\
\text { - NCT01433991 }\end{array}$ & Phase Ib/II & Advanced solid tumors & Ongoing \\
\hline
\end{tabular}


in the midst of searching for an accurate biomarker that could be used to identify patients who would benefit the most from treatment with MET inhibitors. Results from the METIV-HCC trial will help clarify the issue. Given the role of MET in angiogenesis, tissue and serum VEGF levels, and circulating endothelial cells are also being explored as biomarkers of treatment response.

\section{Conclusion}

Many molecular therapies that target signaling cascades involved in hepatocarcinogenesis have been explored in Phase III clinical trials including brivanib, sunitinib, erlotinib and linifanib as first-line treatments, and brivanib and everolimus as second-line treatments after sorafenib failure. However, none of the drugs tested have shown positive results thus far. Although we look forward to receiving encouraging results from ongoing trials of targeted MET therapies, overall, anti-MET therapeutic early clinical trials have demonstrated modest results.

There are several possible explanations for the modest effects observed in patients with HCC. First, this tumor is heterogeneous, owing to the risk factors and the tumor microenvironment, and researchers have yet to take these background factors into consideration in preclinical models. Second, although IHC staining is currently the standard method for identifying HCC patient with MET overexpression who may benefit the most from MET-targeted therapies, there is no definitive evidence that this characteristic predicts treatment response, or that $M E T$ is in fact an oncogenic driver in HCC. Third, in contrast to patients with other solid tumors, most HCC patients have underlying cirrhosis, which makes it difficult to evaluate both the efficacy and safety of targeted therapies since liver function is reduced; therefore, recruiting these patients for clinical trials is challenging.

The ongoing trials of HGF/MET inhibitors in HCC are shown in Table 1. Based on these ongoing prospective studies and future clinical studies, we hope to develop a standardized method for predicting MET dependence. In addition to designing trials based on valid biomarkers and that target the proper patient population, selecting an adequate end point or surrogate marker, along with drugs with potentially significant antitumor activities are needed for the development of MET inhibitors [76]. Although the role of HGF/MET in HCC remains elusive, preclinical and clinical data still indicate that MET is a promising therapeutic target, and more rigorous characterization of the pharmacologic activities of targeted drugs along with well-designed clinical trials that include patients selected based on the activation status of the MET pathway would facilitate unbiased clinical testing thereby providing more benefits to the patients participating in the trials.

\section{Future perspective}

Many molecular therapies that target signaling cascades involved in hepatocarcinogenesis including HGF/MET signaling have been explored in Phase III clinical trials. Hepatocellular carcinoma is a heterogeneous disease owing to the

\section{EXECUTIVE SUMMARY}

\section{Background}

- Hepatocellular carcinoma (HCC) is considered a chemoresistant cancer; therefore, targeted molecular therapies are a highly active area of research.

- The HGFR, a tyrosine kinase encoded by the $\mathrm{N}$-methyl-N'-nitrosoguanidine human osteosarcoma transforming gene $(M E T)$, has been investigated as a possible therapeutic target in HCC.

\section{Role of MET in tumor biology}

- The MET proto-oncogene encodes the tyrosine kinase receptor for HGF. Once activated, the HGF/MET complex signals through the RAS/MAPK and PI3K/AKT pathways thereby promoting several biological activities including cell proliferation, invasion, protection from apoptosis and angiogenesis.

- MET expression has been demonstrated in various solid tumors and has been correlated with poor prognosis.

\section{Agents that target the MET signaling pathway}

- Currently, there are no approved drugs that target the MET signaling pathway in HCC. Several drugs that target the HGF/MET axis are under clinical investigation. These include monoclonal antibodies against HGF or MET, and specific or nonspecific small-molecule MET tyrosine kinase inibitors. 
associated risk factors and tumor microenvironment, and these background factors have yet to be accounted for in preclinical models. IHC staining is currently the standard method for identifying patients with MET overexpression who may benefit the most from MET-targeted therapies; however, a better characterization of the pharmacologic activities of targeted drugs along with well-designed clinical trials is needed.

Financial \& competing interests disclosure

The authors have no relevant affliations or financial involvement with any organization or entity with a financial interest in or financial conflict with the subject matter or materials discussed in the manuscript. This includes employment, consultancies, honoraria, stock ownership or options, expert testimony, grants or patents received or pending, or royalties.

No writing assistance was utilized in the production of this manuscript.

\section{Open access}

This work is licensed under the Creative Commons Attribution-NonCommercial 4.0 Unported License. To view a copy of this license, visit http://creativecommons.org/ licenses/by-nc-nd/4.0/

\section{References}

Papers of special note have been highlighted as: - of interest; $\bullet \bullet$ of considerable interest

1 Parkin DM, Bray F, Ferlay J, Pisani P. Global cancer statistics, 2002. CA Cancer J. Clin. 55(2), 74-108 (2005).

2 El-Serag HB. Hepatocellular carcinoma: recent trends in the United States. Gastroenterology 127(5 Suppl. 1), S27-S34 (2004).

3 Matsuda A, Matsuda T, Shibata A et al. Cancer incidence and incidence rates in Japan in 2008: a study of 25 population-based cancer registries for the Monitoring of Cancer Incidence in Japan (MCIJ) project. Jpn. J. Clin. Oncol. 44(4), 388-396 (2014).

4 Tanaka Y, Kurbanov F, Mano $S$ et al. Molecular tracing of the global hepatitis C virus epidemic predicts regional patterns of hepatocellular carcinoma mortality. Gastroenterology 130(3), 703-714 (2006).

5 El-Serag HB, Hampel H, Javadi F. The association between diabetes and hepatocellular carcinoma: a systematic review of epidemiologic evidence. Clin. Gastroenterol. Hepatol. 4(3), 369-380 (2006).

6 Forner A, Llovet JM, Bruix J. Hepatocellular carcinoma. Lancet 379 (9822), 1245-1255 (2012).

7 Llovet JM, Ricci S, Mazzaferro V et al. Sorafenib in advanced hepatocellular carcinoma. N. Engl. J. Med. 359(4), 378-390 (2008).

8 Giordano S, Ponzetto C, Di Renzo MF, Cooper CS, Comoglio PM. Tyrosine kinase receptor indistinguishable from the c-met protein. Nature 339(6220), 155-156 (1989).

- A protein, p190, indistinguishable from the protein encoded by the MET proto-oncogene was identified, and the high level of p190 found in the cell line was accompanied by amplification and overexpression of MET.
This provided the first example of a functional alteration of MET in a human tumor cell line.

9 Birchmeier C, Birchmeier W, Gherardi E, Vande Woude GF. Met, metastasis, motility and more. Nat. Rev. Mol. Cell Biol. 4(12), 915-925 (2003).

10 Nakamura T, Nishizawa T, Hagiya M et al. Molecular cloning and expression of human hepatocyte growth factor. Nature 342(6248), 440-443 (1989).

11 Schmidt L, Duh FM, Chen F et al. Germline and somatic mutations in the tyrosine kinase domain of the $M E T$ proto-oncogene in papillary renal carcinomas. Nat. Genet. 16(1), 68-73 (1997).

- The identification of missense germline mutations located in the tyrosine kinase domain of the $M E T$ gene in affected members of hereditary papillary renal carcinoma led to further studies of the role of $M E T$ proto-oncogene in other solid tumors.

12 Van Der Voort R, Taher TE, Wielenga VJ et al. Heparan sulfate-modified CD44 promotes hepatocyte growth factor/scatter factor-induced signal transduction through the receptor tyrosine kinase c-Met. J. Biol. Chem. 274(10), 6499-6506 (1999).

13 Trusolino L, Bertotti A, Comoglio PM. A signaling adapter function for alpha6beta4 integrin in the control of HGF-dependent invasive growth. Cell 107(5), 643-654 (2001).

14 Jo M, Stolz DB, Esplen JE, Dorko K, Michalopoulos GK, Strom SC. Cross-talk between epidermal growth factor receptor and c-Met signal pathways in transformed cells. J. Biol. Chem. 275(12), 8806-8811 (2000).

- The increase of MET phosphorylation by TGF- $\alpha$ in cell lines was inhibited by neutralizaing antibodies against EGFR, indicating that MET associates with EGFR in tumor cells in the absence of HGF.

15 Lu KV, Chang JP, Parachoniak CA et al. VEGF inhibits tumor cell invasion and mesenchymal transition through a MET/ VEGFR2 complex. Cancer Cell 22(1), 21-35 (2012).

16 Fischer OM, Giordano S, Comoglio PM, Ullrich A. Reactive oxygen species mediate Met receptor transactivation by $\mathrm{G}$ proteincoupled receptors and the epidermal growth factor receptor in human carcinoma cells. J. Biol. Chem. 279(28), 28970-28978 (2004).

17 Fornari F, Milazzo M, Chieco P et al. MiR-199a-3p regulates mTOR and c-Met to influence the doxorubicin sensitivity of human hepatocarcinoma cells. Cancer Res. 70 (12), 5184-5193 (2010).

18 Ji Q, Hao X, Zhang M et al. MicroRNA miR-34 inhibits human pancreatic cancer tumor-initiating cells. PLoS ONE 4(8), e6816 (2009).

19 Ohta K, Hoshino H, Wang J et al. MicroRNA-93 activates c-Met/PI3K/Akt pathway activity in hepatocellular carcinoma by directly inhibiting PTEN and CDKN1A. Oncotarget 6(5), 3211-3224 (2015).

20 Comoglio PM, Giordano S, Trusolino L. Drug development of MET inhibitors: targeting oncogene addiction and expedience. Nat. Rev. Drug Discov. 7(6), 504-516 (2008).

21 Takanami I, Tanana F, Hashizume T et al. Hepatocyte growth factor and c-Met/ hepatocyte growth factor receptor in pulmonary adenocarcinomas: an evaluation of their expression as prognostic markers. Oncology 53(5), 392-397 (1996).

22 Siegfried JM, Weissfeld LA, Singh-Kaw P, Weyant RJ, Testa JR, Landreneau RJ. Association of immunoreactive hepatocyte growth factor with poor survival in resectable 
non-small cell lung cancer. Cancer Res. 57(3), 433-439 (1997).

23 Engelman JA, Zejnullahu K, Mitsudomi T et al. MET amplification leads to gefitinib resistance in lung cancer by activating ERBB3 signaling. Science 316(5827), 1039-1043 (2007).

24 Schiller Jh AW, Brugger W, Ferrari D et al. Results from ARQ 197-209: a global randomized placebo-controlled Phase II clinical trial of erlotinib plus ARQ 197 versus erlotinib plus placebo in previously treated EGFR inhibitor-naive patients with locally advanced or metastatic non-small cell lung cancer (NSCLC). J. Clin. Oncol. 28(18s), Abstract LBA7502 (2010).

25 Scagliotti GV, Novello S, Schiller JH et al. Rationale and design of MARQUEE: a Phase III, randomized, double-blind study of tivantinib plus erlotinib versus placebo plus erlotinib in previously treated patients with locally advanced or metastatic, nonsquamous, non-small-cell lung cancer. Clin. Lung Cancer 13(5), 391-395 (2012).

26 Bessudo A, Gabrail N., Kopp MV et al. Phase I results of the randomized, placebo controlled, Phase I/II study of the novel oral c-MET inhibitor, ARQ 197, irinotecan (CPT-11), and cetuximab (C) in patients (pts) with wild-type (WT) KRAS metastatic colorectal cancer (mCRC) who have received front-line systemic therapy. J. Clin. Oncol. 29(Suppl.), Abstract 3582 (2011).

27 Kosai K, Matsumoto K, Nagata S, Tsujimoto Y, Nakamura T. Abrogation of Fas-induced fulminant hepatic failure in mice by hepatocyte growth factor. Biochem. Biophys. Res. Commun. 244(3), 683-690 (1998).

28 Aberger F, Schmidt G, Richter K. The Xenopus homologue of hepatocyte growth factor-like protein is specifically expressed in the presumptive neural plate during gastrulation. Mech. Dev. 54(1), 23-37 (1996).

29 Takami T, Kaposi-Novak P, Uchida K et al. Loss of hepatocyte growth factor/c-Met signaling pathway accelerates early stages of $\mathrm{N}$-nitrosodiethylamine induced hepatocarcinogenesis. Cancer Res. 67(20), 9844-9851 (2007).

30 Marx-Stoelting P, Borowiak M, Knorpp T, Birchmeier C, Buchmann A, Schwarz M. Hepatocarcinogenesis in mice with a conditional knockout of the hepatocyte growth factor receptor c-Met. Int. J. Cancer 124(8), 1767-1772 (2009).

31 Tward AD, Jones KD, Yant S, Kay MA, Wang $\mathrm{R}$, Bishop JM. Genomic progression in mouse models for liver tumors. Cold Spring Harb. Symp. Quant. Biol. 70, 217-224 (2005).
32 Zhang SZ, Pan FY, Xu JF et al. Knockdown of c-Met by adenovirus-delivered small interfering RNA inhibits hepatocellular carcinoma growth in vitro and in vivo. Mol. Cancer Ther. 4(10), 1577-1584 (2005).

33 Salvi A, Arici B, Portolani N, Giulini SM, De Petro G, Barlati S. In vitro c-met inhibition by antisense RNA and plasmid-based RNAi down-modulates migration and invasion of hepatocellular carcinoma cells. Int. J. Oncol. 31(2), 451-460 (2007).

34 Xie B, Xing R, Chen P et al. Down-regulation of c-Met expression inhibits human HCC cells growth and invasion by RNA interference. J. Surg. Res. 162(2), 231-238 (2010).

35 Sakata H, Takayama H, Sharp R, Rubin JS, Merlino G, Larochelle WJ. Hepatocyte growth factor/scatter factor overexpression induces growth, abnormal development, and tumor formation in transgenic mouse livers. Cell Growth Differ. 7(11), 1513-1523 (1996).

36 Horiguchi $\mathrm{N}$, Takayama $\mathrm{H}$, Toyoda $\mathrm{M}$ et al. Hepatocyte growth factor promotes hepatocarcinogenesis through c-Met autocrine activation and enhanced angiogenesis in transgenic mice treated with diethylnitrosamine. Oncogene 21(12), 1791-1799 (2002).

37 Shiota G, Wang TC, Nakamura T, Schmidt EV. Hepatocyte growth factor in transgenic mice: effects on hepatocyte growth, liver regeneration and gene expression. Hepatology 19(4), 962-972 (1994).

38 Shiota G, Rhoads DB, Wang TC, Nakamura T, Schmidt EV. Hepatocyte growth factor inhibits growth of hepatocellular carcinoma cells. Proc. Natl Acad. Sci. USA 89(1), 373-377 (1992).

39 Ogasawara H, Hiramoto J, Takahashi M et al. Hepatocyte growth factor stimulates DNA synthesis in rat preneoplastic hepatocytes but not in liver carcinoma cells. Gastroenterology 114(4), 775-781 (1998).

40 Giordano S, Columbano A. Met as a therapeutic target in HCC: facts and hopes. J. Hepatol. 60 (2), 442-452 (2014).

41 Suzuki M, Shiraha H, Fujikawa T et al. Des-gamma-carboxy prothrombin is a potential autologous growth factor for hepatocellular carcinoma. J. Biol. Chem. 280(8), 6409-6415 (2005).

42 Kondo S, Ojima $\mathrm{H}$, Tsuda $\mathrm{H}$ et al. Clinical impact of c-Met expression and its gene amplification in hepatocellular carcinoma. Int. J. Clin. Oncol. 18(2), 207-213 (2013).

43 Wang K, Lim HY, Shi S et al. Genomic landscape of copy number aberrations enables the identification of oncogenic drivers in hepatocellular carcinoma. Hepatology 58(2), 706-717 (2013).

44 Guichard C, Amaddeo G, Imbeaud S et al. Integrated analysis of somatic mutations and focal copy-number changes identifies key genes and pathways in hepatocellular carcinoma. Nat. Genet. 44(6), 694-698 (2012).

45 Suzuki K, Hayashi N, Yamada Y et al. Expression of the c-met protooncogene in human hepatocellular carcinoma. Hepatology 20(5), 1231-1236 (1994).

46 Noguchi O, Enomoto N, Ikeda T, Kobayashi F, Marumo F, Sato C. Gene expressions of c-met and hepatocyte growth factor in chronic liver disease and hepatocellular carcinoma. J. Hepatol. 24(3), 286-292 (1996).

47 Ueki T, Fujimoto J, Suzuki T, Yamamoto H, Okamoto E. Expression of hepatocyte growth factor and its receptor c-met proto-oncogene in hepatocellular carcinoma. Hepatology 25(4), 862-866 (1997).

48 Vejchapipat P, Tangkijvanich P, Theamboonlers A, Chongsrisawat V, Chittmittrapap S, Poovorawan Y. Association between serum hepatocyte growth factor and survival in untreated hepatocellular carcinoma. J. Gastroenterol. 39(12), 1182-1188 (2004).

49 Schiering N, Knapp S, Marconi Met al. Crystal structure of the tyrosine kinase domain of the hepatocyte growth factor receptor c-Met and its complex with the microbial alkaloid K-252a. Proc. Natl Acad. Sci. USA 100(22), 12654-12659 (2003).

50 Sattler M, Pride YB, Ma P et al. A novel small molecule met inhibitor induces apoptosis in cells transformed by the oncogenic TPRMET tyrosine kinase. Cancer Res. 63(17), 5462-5469 (2003).

51 Christensen JG, Schreck R, Burrows J et al. A selective small molecule inhibitor of c-Met kinase inhibits c-Met-dependent phenotypes in vitro and exhibits cytoreductive antitumor activity in vivo. Cancer Res. 63(21), 7345-7355 (2003).

52 You H, Ding W, Dang H, Jiang Y, Rountree CB. c-Met represents a potential therapeutic target for personalized treatment in hepatocellular carcinoma. Hepatology 54(3), 879-889 (2011).

53 Yau TC, Chao Y, Yen CJ et al. A Phase I/II study of foretinib, an oral multikinase inhibitor targeting MET, RON, AXL, TIE-2, and VEGFR in advanced hepatocellular carcinoma (HCC). J. Clin. Oncol. 30 (Suppl.), Abstract 4108 (2012). 
54 Verslype C, Kelley RK, Yang TS et al. Activity of cabozantinib (XL184) in hepatocellular carcinoma: Results from a Phase II randomized discontinuation trial (RDT). J. Clin. Oncol. 30(Suppl.), Abstract 4007 (2012).

55 Goldman JW, Rosen LS, Algazi AP et al. First-in-human dose escalation study of LY2875358 (LY), a bivalent MET antibody, as monotherapy and in combination with erlotinib (E) in patients with advanced cancer. J. Clin. Oncol. 31(Suppl.), Abstract 8093 (2013).

56 Munshi N, Jeay S, Li Y et al. ARQ 197, a novel and selective inhibitor of the human c-Met receptor tyrosine kinase with antitumor activity. Mol. Cancer Ther. 9(6), 1544-1553 (2010).

57 Yap TA, Olmos D, Brunetto AT et al. Phase I trial of a selective c-MET inhibitor ARQ 197 incorporating proof of mechanism pharmacodynamic studies. J. Clin. Oncol. 29(10), 1271-1279 (2011).

- This Phase I study of 51 patients with tivantinib included 15 patients who underwent pair biopsies and identified biomarker expression differences of phosphorylated c-MET and total c-MET $(\mathrm{p}=0.041)$, phosphorylated FAK $(\mathrm{p}=0.042)$ and TUNEL ( $\mathrm{p}=0.011$ ) between pretherapy and on therapy tumors.

58 Santoro A, Rimassa L, Borbath I et al. Tivantinib for second-line treatment of advanced hepatocellular carcinoma: a randomised, placebo-controlled Phase 2 study. Lancet Oncol. 14(1), 55-63 (2013).

59 Katayama R, Aoyama A, Yamori T et al. Cytotoxic activity of tivantinib (ARQ 197) is not due solely to c-MET inhibition. Cancer Res. 73(10), 3087-3096 (2013).

60 Basilico C, Pennacchietti S, Vigna E et al. Tivantinib (ARQ197) displays cytotoxic activity that is independent of its ability to bind MET. Clin. Cancer. Res. 19(9), 2381-2392 (2013).

61 Okusaka T, Ikeda M, Fukutomi A et al. Response to Y. Sasaki et al.: is repeating FOLFIRINOX in the original dosage and treatment schedule tolerable in Japanese patients with pancreatic cancer? Cancer Sci. 106(8), 1101-1102 (2015).
62 Bang YJ, Nam DH, Lim WT et al. Phase I study of the safety and efficacy of INC280 in patients with advanced MET-dependent solid tumors. J. Clin. Oncol. 32(Suppl. 5s), Abstract 2520 (2014).

63 Falchook Gs HD, Amin Hm, Fu S et al. Phase I study of oral selective c-Met inhibitor EMD 1214063 in pts with advanced solid tumors. J. Clin. Oncol. 31(Suppl.), Abstract 2506 (2013).

64 Bussolino F, Di Renzo MF, Ziche M et al. Hepatocyte growth factor is a potent angiogenic factor which stimulates endothelial cell motility and growth. J. Cell Biol. 119(3), 629-641 (1992).

65 Eckerich C, Zapf S, Fillbrandt R, Loges S, Westphal M, Lamszus K. Hypoxia can induce c-Met expression in glioma cells and enhance SF/HGF-induced cell migration. Int. J. Cancer 121(2), 276-283 (2007).

66 Pennacchietti S, Michieli P, Galluzzo M, Mazzone M, Giordano S, Comoglio PM. Hypoxia promotes invasive growth by transcriptional activation of the met protooncogene. Cancer Cell 3(4), 347-361 (2003).

67 Puzanov I, Sosman J, Santoro A et al. Phase 1 trial of tivantinib in combination with sorafenib in adult patients with advanced solid tumors. Invest. New Drugs 33(1), 159-168 (2015).

- Twenty hepatocellular carcinoma patients in this Phase I study of tivantinib plus sorafenib resulted in response rate of $10 \%$, including patients who progressed on sorafenib who obtained partial responses and one complete response. This demonstrated the clinical benefit of the addition of MET inhibition for those who acquired resistance to anti-VEGFR agents.

68 O'neil BH, Modiano MR, Machiels JPH et al. Phase I/II study of E7050 (golvantinib) in combination with sorafenib in patients (pts) with advanced hepatocellular carcinoma (HCC): Phase I results. J. Clin. Oncol. 31(Suppl. 4), Abstract 294 (2013).

69 Nakazawa Y, Kawano S, Matsui J et al. Multitargeting strategy using lenvatinib and golvatinib: maximizing anti-angiogenesis activity in a preclinical cancer model. Cancer Sci. 106(2), 201-207 (2015).
70 Scagliotti G, Von Pawel J, Novello S et al. Phase III multinational, randomized, double-blind, placebo-controlled study of tivantinib (ARQ 197) plus erlotinib versus erlotinib alone in previously treated patients with locally advanced or metastatic nonsquamous non-small-cell lung cancer. J. Clin. Oncol. 33(24), 2667-2674 (2015).

- In non-small-cell lung cancer population treated with tivantinib and erlotinib, subgroup analyses suggested overall survival improvement in patients with high MET expression (HR: 0.70; 95\% CI: 0.49-1.01) and progression-free survival increase (median progression-free survival: $3.6 \mathrm{vs}$ 1.9 months; HR: 0.74; 95\% CI: 0.62-0.89; $\mathrm{p}<0.001)$

71 Eng C HL, Severtsev a, Gladkov O et al. A randomized, placebo-controlled, Phase I/II study of tivantinib (ARQ 197) in combination with cetuximab and irinotecan in patients (pts) with KRAS wild-type (WT) metastatic colorectal cancer (CRC) who had received previous front-line systemic therapy. J. Clin. Oncol. 31(Suppl. 15), Abstract 3508 (2013).

72 Liu X, Shi Y, Maag DX et al. Iniparib nonselectively modifies cysteine-containing proteins in tumor cells and is not a bona fide PARP inhibitor. Clin. Cancer Res. 18(2), 510-523 (2012).

73 Patel AG, De Lorenzo SB, Flatten KS, Poirier GG, Kaufmann SH. Failure of iniparib to inhibit poly(ADP-Ribose) polymerase in vitro. Clin. Cancer Res. 18(6), 1655-1662 (2012).

74 Previdi S, Abbadessa G, Dalo F, France DS, Broggini M. Breast cancer-derived bone metastasis can be effectively reduced through specific c-MET inhibitor tivantinib (ARQ 197) and shRNA c-MET knockdown. Mol. Cancer Ther. 11(1), 214-223 (2012).

$75 \mathrm{Lu}$ S, Torok HP, Gallmeier E et al. Tivantinib (ARQ 197) affects the apoptotic and proliferative machinery downstream of c-MET: role of Mcl-1, Bcl-xl and cyclin B1. Oncotarget 6(26), 22167-22178 (2015).

76 Llovet JM, Hernandez-Gea V. Hepatocellular carcinoma: reasons for Phase III failure and novel perspectives on trial design. Clin. Cancer Res. 20 (8), 2072-2079 (2014). 